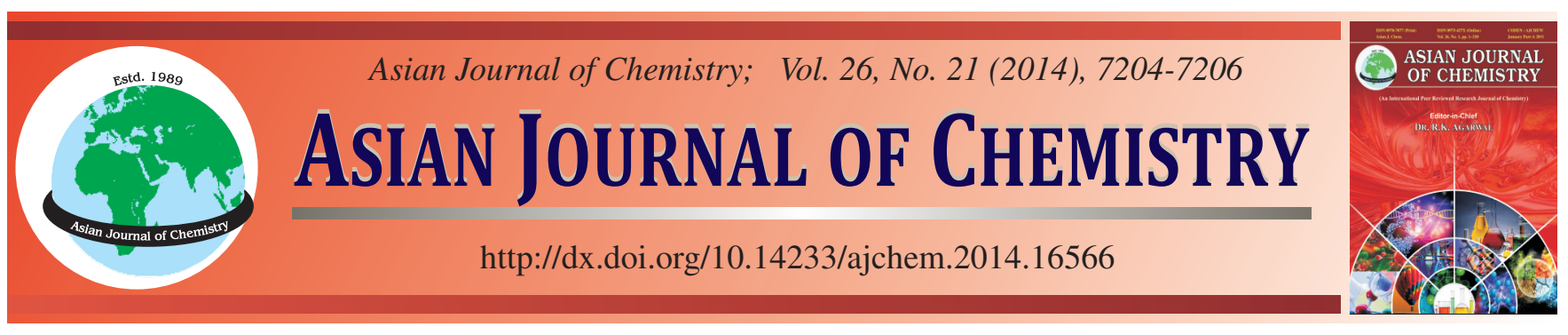

\title{
Preparation of Mesoporous Carbon Using Phenol-Formaldehyde Resin as Carbon Precursor and Ethylene Glycol as Pore-Forming Agent
}

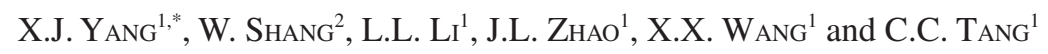

${ }^{1}$ School of Materials Science and Engineering, Hebei University of Technology, Tianjin 300130, P.R. China

${ }^{2}$ Institute of Engineering Mechanics, Tianjin Chengjian University, Tianjin 300384, P.R. China

*Corresponding author: Tel/Fax: +86 22 60204805; E-mail: yxjingtssw@gmail.com; yxjing@mail.nankai.edu.cn

We reported a convenient route to synthesize mesoporous carbon. The carbon was prepared in a mixture of phenol-formaldehyde resin (PF) as a carbon precursor and ethylene glycol (EG) as a pore-forming agent. The structure, morphology and the specific surface area of the obtained samples were characterized by X-ray diffraction, scanning electron microscopy and Quadrasorb SI sorption analyzer. The results show that the diameters of the mesoporous carbon are about $20-30 \mathrm{~nm}$. It is found that the specific surface areas of the product are changed with the ratios of the value for PF/EG. The porous carbon exhibits the highest specific surface area $\left(175.3 \mathrm{~m}^{2} / \mathrm{g}\right)$ when $\mathrm{PF} / \mathrm{EG}$ is $3: 7$.

Keywords: Mesoporous carbon, Microstructure, Phenol resin.

\section{INTRODUCTION}

Porous carbon materials have received a great deal of attention due to their applications ${ }^{1}$. Porous carbon materials have been applied to gas separation, water purification, catalyst supports and electrodes for fuel cells ${ }^{2-5}$. Porous carbon materials have been synthesized using various methods, such as physical and chemical activation ${ }^{6}$, catalytic activation ${ }^{7}$ and carbonization processes ${ }^{8}$. Many kinds of materials, such as starch, cyclodextrin, coal and resins, are employed as carbonaceous material to prepare mesoporous carbons ${ }^{9-11}$.

Phenol resins are increasingly used as precursors for various carbons in the form of fibers, particles and membranes ${ }^{12-14}$. Phenol resins is used to prepare glassy carbon (GC) by pyrolyzing under inert atmosphere and temperatures between 600 and $3000{ }^{\circ} \mathrm{C}^{15}$. Usually, glassy carbons contain a high proportion of closed pores that are not accessible for gas molecules. So it is ineffective in their applications. Physico-chemical activation and pyrolyzed are two effective methods to open these closed pores. In activation method, phenol resin is usually first carbonized to obtain the pyrolytic material, which is then modified. However, integrated monolithic porous carbons are difficult to obtain with above two methods ${ }^{9,11}$.

In this work, ethylene glycol (EG) is employed as a poreforming agent to form opened pores in carbonized materials derived from phenol-formaldehyde resins (PF). And the influence of various mass ratio of phenol-formaldehyde and ethylene glycol on the pore structure of porous carbons obtained is investigated.

\section{EXPERIMENTAL}

All the reagents were of analytical grade and used without further purification. The as-synthesized catalysts were characterized by powder X-ray diffraction (XRD, Rigaku D/max$2500 \mathrm{X}$-ray generator, $\mathrm{CuK}_{\alpha}$ radiation), scanning electron microscopy (SEM, JEOL JSM-6700F), nitrogen adsorptiondesorption isotherms were carried out at $77.3 \mathrm{~K}$ on a Quadrasorb SI sorption analyzer. The samples were outgassed for $8 \mathrm{~h}$ at $300{ }^{\circ} \mathrm{C}$ under a vacuum in the degasport of the analyzer. The specific surface area was calculated with the Brunauer-EmmettTeller (BET) model and pore size distribution was calculated from the adsorption-desorption data using the Density Functional Theory (DFT) method.

General procedure: In a typical procedure, phenol-formaldehyde resin (PF) was employed as carbon precursor. Ethylene glycol (EG) served as solvent and pore-forming agent and benzenesulfonyl chloride was used as a curing catalyst. Phenol resin, ethylene glycol and benzenesulfonyl chloride were mixed by stirring mechanically for $0.5 \mathrm{~h}$ at room temperature, then poured into molds and cured at $50{ }^{\circ} \mathrm{C}$ for $6 \mathrm{~h}$. After $6 \mathrm{~h}$, the rigid body was removed from mold while excess glycol was removed by draining with absorbent paper. The rigid body was then postcured by less than $2{ }^{\circ} \mathrm{C} / \mathrm{h}$ velocity to $180{ }^{\circ} \mathrm{C}$ 
from $60{ }^{\circ} \mathrm{C}$ and held for $16 \mathrm{~h}$. The sample was pyrolyzed by slowly ramping to $800{ }^{\circ} \mathrm{C}$ in a flowing nitrogen atmosphere, followed by cooling naturally to room temperature. The mass ratio of phenol-formaldehyde and ethylene glycol was 7:3, $1: 1,2: 3$ and 3:7, respectively. And the resulting porous carbon was designated as S-1, S-2, S-3 and S-4, accordingly.

\section{RESULTS AND DISCUSSION}

In XRD pattern of the synthesized carbon simple. Two characteristic diffraction peaks in the curves can be indexed as (002) and (100) crystal planes of carbon. The diffraction peak at $2 \theta$ value of $23.2^{\circ}$ corresponds to the (002) plane and the $d_{002}$ is $0.3832 \mathrm{~nm}$ measured by the width of the peak at $23.2^{\circ}$.

The morphology of the obtained products was investigated by SEM analysis. Fig. 1 shows the SEM images of the obtained S-1 to S-4 samples. Fig. 1 illustrates the micrographs of porous carbons prepared with different $\mathrm{PF} / \mathrm{EG}$ ratio. It is obvious that the morphologies of the carbons obtained show differences. S-1 and S-2 are all nanoparticles and well distributed with diameter of about several nanometers. A large number of interconnected pores with pore size of nanometer are observed in S-3 and S-4 (Fig. 1c and d).

More information from Fig. 1c and d can be proved by the Fig. 2. The size distribution of the samples is shown in the diameter histograms (Fig. 2) obtained by statistically measuring the sizes of more than 50 individual pores on SEM images. The pores of S-3 are well distributed with an average diameter of about $25 \mathrm{~nm}$ (Fig. 2a), while S-4 possesses the average pore size of $30 \mathrm{~nm}$ (Fig. 2b). The decreasing of the PF/EG ratio results in different sizes of pore.

Fig. 3 shows the nitrogen adsorption/desorption isotherms and pore size distribution for S-1 to S-4 obtained from an automatic adsorption instrument. The desorption and adsorption curves of S-1 and S-2 are almost coincide with each other, respectively. This means that the samples of S-1 and $\mathrm{S}-2$ have no mesoporous. It is obvious that the samples of S-3 and S-4 exhibit type IV isotherms, corresponds to mesoporous adsorption ${ }^{16}$. Characteristic features of the isotherms are its hysteresis loop, which is associated with capillary condensation taking place in mesopores and the limiting uptake over a range of high $\mathrm{P} / \mathrm{P}^{\circ}$. The initial part of the isotherm is attributed to monolayer-multilayer adsorption.

The pore size distribution of S-3 and S-4 from nitrogen adsorption/desorption isotherm data agrees basically with that deduced from the results of SEM. The pores are mainly located at 20 to $30 \mathrm{~nm}$ and the pore sizes distribute narrowly. It is attributed to the carbon skeleton. Small peaks before $20 \mathrm{~nm}$ for S-3 and S-4 can be attributed to the stacked pore.

Table-1 lists the data of pore sizes and specific surface areas of S-1 to S-4 shown in the Figs. 3 and 4. It shows the average pore sizes increased from 22.9 to $30.8 \mathrm{~nm}$ with decreasing the ratio of PF/EG. The BET surface area of carbons has continuously increased with the decrease of PF/EG ratios. When the mass ratio of PF and EG is 2:3, the specific surface area reached the maximal value $\left(175.3 \mathrm{~m}^{2} / \mathrm{g}\right)$. It can be drawn a conclusion that S-3 and S-4 are confirmed to be mesoporous carbons from the data shown in Table-1, Figs. 3 and 4.
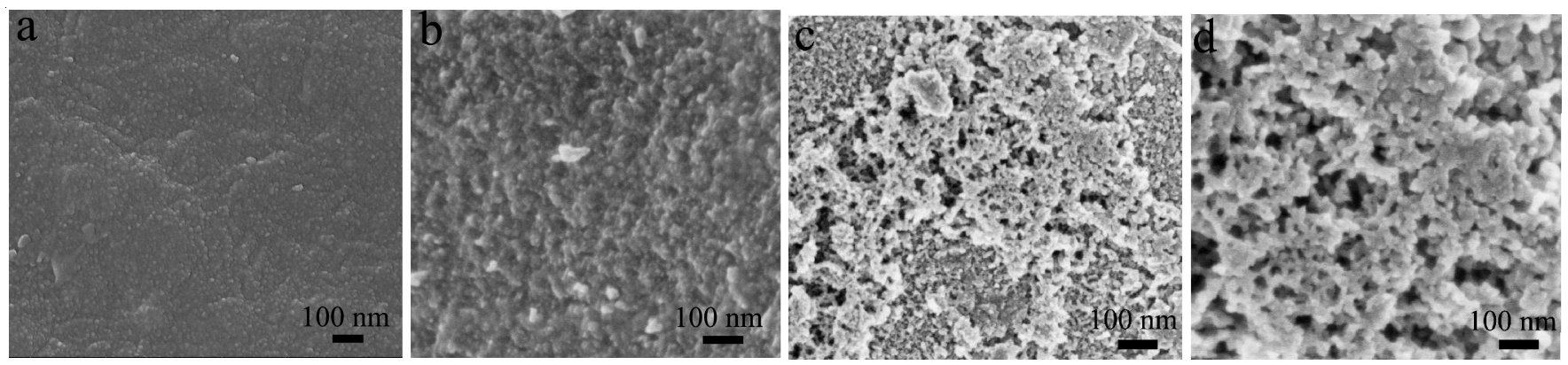

Fig. 1. SEM imagin of the product: (a) S-1, (b) S-2, (c) S-3, (d) S-4
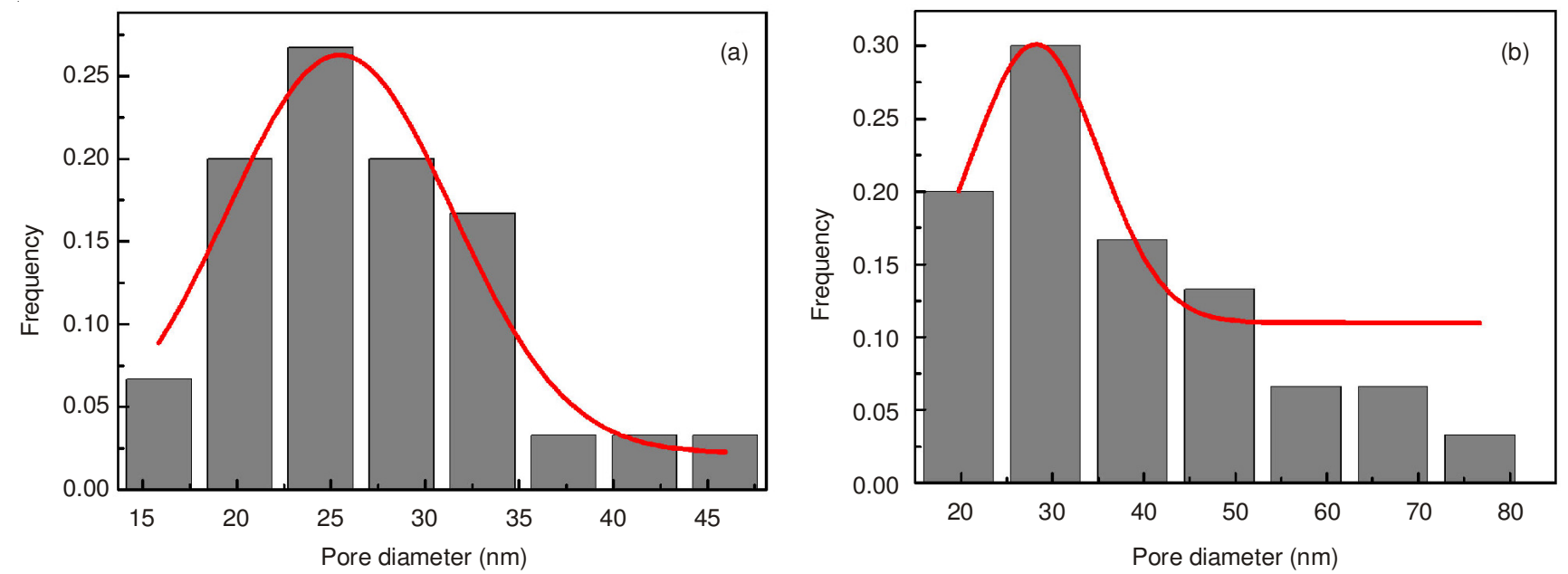

Fig. 2. Pore size distributions of the product: (a) S-3, (b) S-4 


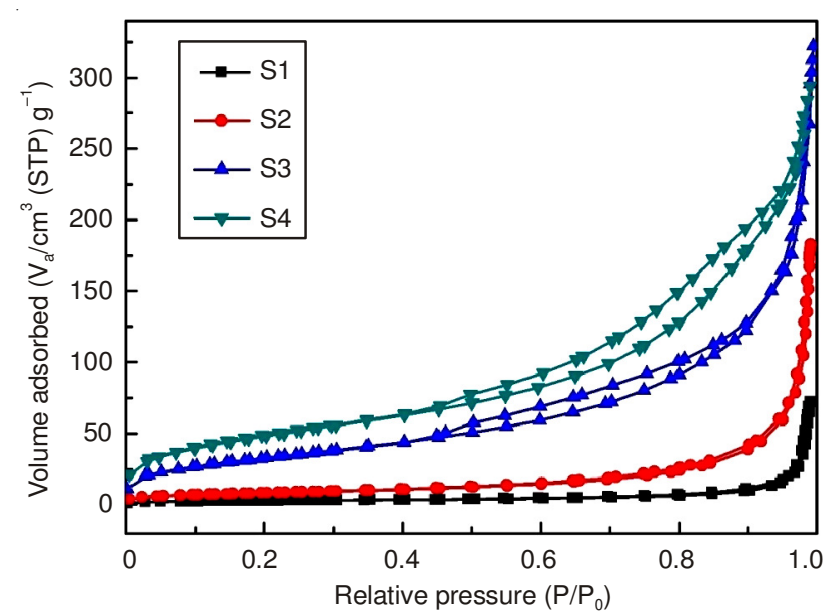

Fig. 3. $\mathrm{N}_{2}$ adsorption/desorption isotherms of S-1 to S-4

\begin{tabular}{cccc}
\multicolumn{4}{c}{ TABLE-1 } \\
\multicolumn{4}{c}{ PROPERTIES OF S-1 TO S-4 } \\
\hline Simple & PF/EG & Pore size $(\mathrm{nm})$ & specific surface area $\left(\mathrm{m}^{2} / \mathrm{g}\right)$ \\
\hline S-1 & $7: 3$ & - & 9.615 \\
S-2 & $1: 1$ & - & 29.64 \\
S-3 & $2: 3$ & 22.9 & 121.1 \\
S-4 & $3: 7$ & 30.8 & 175.3 \\
\hline
\end{tabular}

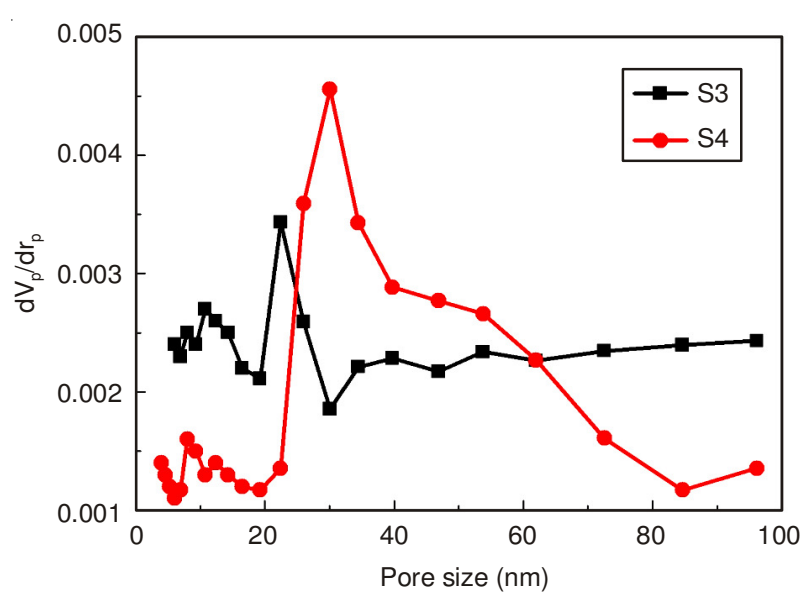

Fig. 4. Pore size distribution of S-3 and S-4

The above results indicate that the pores of the synthesized carbon are due to the rates of PF/EG. The porous carbon exhibits the highest specific surface area when PF/EG is 3:7. The miscibility of polymeric resin in EG is decreasing with the increasing of molecular weight and then phase separation in the resin mixture develops concurrently with polymerization, with only a polymeric resin-rich phase and a glycol-rich phase being obviously. After postcuring and subsequent pyrolysis, the former becomes a carbon skeleton, while the latter is removed and results in pores ${ }^{17}$. During phase separation with various $\mathrm{PF} / \mathrm{EG}$ ratio, dispersed glycol-rich phase is formed as PF/EG $=7 / 3$, while continuous glycol-rich phase is formed as PF/EG $<1$. Because there are no effective channels in the rigid body of subsequently removing the dispersed glycol-phase, sponge structure and some cracks are formed. In contrast, continuous glycol-rich phase in the rigid body endows effective channels for removing of the ethylene glycol, so mesoporous carbons with interconnected pores are obtained ${ }^{18}$.

\section{Conclusion}

In summary, porous carbons have been synthesized via the mixtures of phenol-formaldehyde resin (PF) as a carbon precursor and ethylene glycol (EG) as a pore-forming agent. Furthermore, mesoporous carbons with interconnected pores and apparent porosity were obtained when the $\mathrm{PF} / \mathrm{EG}$ ratio was not more than 1 . Variation in the $\mathrm{PF} / \mathrm{EG}$ ratio caused different morphologies, pore size distribution and the BET surface area of the mesopores carbons. Decreasing the PF/EG ratio endowed the mesoporous carbons with narrow pore size distribution and short average pore size. The average pore size was controlled between 22.9 and $30.8 \mathrm{~nm}$. The specific surface area was $175.3 \mathrm{~m}^{2} / \mathrm{g}$ when the mass ratio of PF/EG was 3:7.

\section{ACKNOWLEDGEMENTS}

This work was supported by the National Natural Science Foundation of China (No. 51301057 and 51272064), Natural Science Foundation of Hebei Province (No. B2013202211 and E2013202032), Open Project of Key Lab Advanced Energy Material Chemistry (Nankai University) (KLAEMCOP201101, KLAEMC-OP201201) and the Key Basic Research Program of Hebei Province of China (No. 12965135D).

\section{REFERENCES}

1. S. Subramoney, Adv. Mater., 10, 1157 (1998).

2. A. Parker, M. Marszewski and M. Jaroniec, Mater. Interfaces, 5, 1948 (2013).

3. X.Y. Shen, D.B. Mu, S. Chen, B.R. Wu and F. Wu, Mater. Interfaces, 5, 3118 (2013).

4. T. Kyotani, Carbon, 38, 269 (2000).

5. Y.G. Wang, Z.H. Min, M. Cao and D.P. Xu, New Carbon Mater, 24, 321 (2009).

6. T.Y. Zhang, W.P. Walawender, L.T. Fan, M. Fan, D. Daugaard and R.C. Brown, Chem. Eng. J., 105, 53 (2004).

7. H. Tamai, T. Kakii, Y. Hirota, T. Kumamoto and H. Yasuda, Chem. Mater., 8, 454 (1996).

8. N. Patel, K. Okabe and A. Oya, Carbon, 40, 315 (2002).

9. H. Teng and S.C. Wang, Carbon, 38, 817 (2000).

10. M.C. Huang and H. Teng, Carbon, 40, 955 (2002).

11. T. Horikawa, K. Ogawa, K. Mizuno, J. Hayashi and K. Muroyama, Carbon, 41, 465 (2003).

12. J. Wu and D.D.L. Chung, Carbon, 40, 445 (2002).

13. J. Ozaki, N. Endo, W. Ohizumi, K. Igarashi, M. Nakahara, A. Oya, S. Yoshida and T. Iizuka, Carbon, 35, 1031 (1997).

14. H. Wang, X. Li, L.Y. Hong and D.-P. Kim, J. Porous Mater., 13, 115 (2006).

15. A. Braun, M. Bärtsch, B. Schnyder, R. Kötz, O. Haas, H.G. Haubold and G. Goerigk, J. Non-Cryst. Solids, 260, 1 (1999).

16. K.S.W. Sing, D.H. Everett, R.A.W. Haul, L. Moscou, R.A. Pierotti and J. Rouquerol, Pure Appl. Chem., 57, 603 (1985).

17. S.J. Xu, G.J. Qiao, H.J. Wang, D.C. Li and T.J. Lu, Mater. Lett., 62, 3716 (2008).

18. X.Y. Zhang, H.Q. Hu, Y.D. Zhu and S.W. Zhu, J. Membr. Sci., 289, 86 (2007). 